See discussions, stats, and author profiles for this publication at: https://www.researchgate.net/publication/267898723

\title{
An integrated genomic-transcriptomic approach supports a role for the proto-oncogene BCL3 in atherosclerosis
}

Article in Thrombosis and Haemostasis · November 2014

DOI: 10.1160/TH14-05-0466

\section{CITATIONS}

10

18 authors, including:

\section{Giovanna Marchetti}

University of Ferrara

143 PUBLICATIONS 2,332 CITATIONS

SEE PROFILE

Carlotta Zerbinati

University of Ferrara

36 PUBLICATIONS 647 CITATIONS

SEE PROFILE

Some of the authors of this publication are also working on these related projects:

Development of LMTK3 inhibitors View project

Project carotid disease View project
191

Domenico Girelli

University of Verona

406 PUBLICATIONS 20,968 CITATIONS

SEE PROFILE

Barbara Lunghi

University of Ferrara

70 PUBLICATIONS 2,178 CITATIONS

SEE PROFILE 


\title{
An integrated genomic-transcriptomic approach supports a role for the proto-oncogene BCL3 in atherosclerosis
}

\author{
Giovanna Marchetti'; Domenico Girelli2; Carlotta Zerbinati'; Barbara Lunghiं ${ }^{3}$ Simonetta Friso²; Silvia Meneghetti'; Matteo Coen \\ Teresa Gagliano ${ }^{5}$;Giuseppe Guastella'; Marie-Luce Bochaton-Piallat ${ }^{4}$; Francesca Pizzolo²; Francesco Mascoli6; Giovanni Malerba7; \\ Matteo Bovolenta ${ }^{3}$; Manuela Ferracin ${ }^{8,9}$; Oliviero Olivieri $^{2}$; Francesco Bernardi ${ }^{3}$; Nicola Martinelli ${ }^{2}$ \\ ${ }^{1}$ Department of Biomedical and Specialty Surgical Sciences, University of Ferrara, Ferrara, Italy; ${ }^{2}$ Department of Medicine, University of Verona, Verona, Italy; ${ }^{3}$ Department of Life \\ Sciences and Biotechnology, University of Ferrara, Ferrara, Italy; ${ }^{4}$ Department of Pathology and Immunology, University of Geneva, Geneva, Switzerland; ${ }^{5}$ Department of Medical \\ Sciences, University of Ferrara, Ferrara, Italy; ${ }^{6}$ Unit of Vascular and Endovascular Surgery, S. Anna University-Hospital, Ferrara, Italy; ${ }^{7}$ Department of Life and Reproduction Sciences, \\ Section of Biology and Genetics, University of Verona, Verona, Italy; ${ }^{8}$ Department of Morphology, Surgery and Experimental Medicine, University of Ferrara, Ferrara, Italy; \\ 'Laboratory for Technologies of Advanced Therapies (LTTA), University of Ferrara, Ferrara, Italy
}

\begin{abstract}
Summary
Data with border-line statistical significance, copiously generated in genome-wide association studies of coronary artery disease (CAD), could include functionally relevant associations. We propose an integrated genomic and transcriptomic approach for unravelling new potential genetic signatures of atherosclerosis. Fifteen among 91 single nucleotide polymorphisms (SNPs) were first selected for association in a sex- and age-adjusted model by examining 510 patients with $C A D$ and myocardial infarction and 388 subjects with normal coronary arteries (CAD-free) in the replication stages of a genome-wide association study. We investigated the expression of 71 genes proximal to the 15 tag-SNPs by two subsequent steps of microarray-based mRNA profiling, the former in vascular smooth muscle cell populations, isolated from non-atherosclerotic and atherosclerotic human carotid portions, and the latter in whole carotid specimens. BCL3 and PVRL2, contiguously located on chromosome 19, and ABCA1, extensively investigated before, were found to be differentially expressed. BCL3 and
\end{abstract}

Correspondence to:

Nicola Martinelli

Department of Medicine, University of Verona

37134 Verona, Italy

E-mail: nicola.martinelli@univr.it
PVRL2 SNPs were genotyped within a second population of CAD patients $(n=442)$ and compared with CAD-free subjects $(n=393)$. The carriership of the BCL3 rs2965169 G allele was more represented among CAD patients and remained independently associated with CAD after adjustment for all the traditional cardiovascular risk factors (odds ratio $=1.70$ with $95 \%$ confidence interval $1.07-2.71$ ), while the $B C L 3$ rs8100239 A allele correlated with metabolic abnormalities. The upregulation of BCL3 mRNA levels in atherosclerotic tissue samples was consistent with $\mathrm{BCL} 3$ protein expression, which was detected by immunostaining in the intima-media of atherosclerotic specimens, but not within non-atherosclerotic ones. Our integrated approach suggests a role for BCL3 in cardiovascular diseases.

\section{Keywords}

$B C L 3$, genomics, transcriptomics, coronary artery disease, metabolic disorders
Financial support:

This study was supported by grants from the Italian Ministry of University and Research; the Veneto Region; the Cariverona Foundation, Verona; the Carife Foundation, Ferrara; the Italian Ministry of Health (Finalized Research "Emilia Romagna Region").

Received: May 26, 2014

Accepted after major revision: October 8, 2010

Epub ahead of print: November 6, 2014

http://dx.doi.org/10.1160/TH14-05-0466

Thromb Haemost 2015; 113: an

\section{Introduction}

Cardiovascular disease (CVD) is a leading health problem in the world, and inherited DNA sequence variants are well recognised to influence CVD risk $(1,2)$. Genome-wide association studies (GWAS) have identified a large number of CVD-associated loci (3, 4) with several of them containing genes not previously implicated in the traditional pathways of CVD. Functional explanations are still lacking for the majority of gene variants identified by GWAS, but required to elucidate the molecular mechanisms with potential application in clinical practice (5-11). Moreover, it is worthy to note that in spite of the abundance of results obtained by GWAS the identified loci explain only a limited proportion of the whole CVD heritability (1). The stringent level of statistical significance required in GWAS may lead to discard genetic variants that could contribute to disease risk (12). Recent observations suggest that GWAS databases likely include a number of "hidden" effective variants that up to now have only suggestive statistical evidence of association $(13,14)$.

Atherosclerosis is the common ground of several clinical manifestations of CVD, including coronary artery disease (CAD), myocardial infarction (MI), peripheral artery occlusive diseases, and 
stroke $(15,16)$. Vascular smooth muscle cells (VSMCs), which are the only cell type of the media layer of normal vessel wall, play also a pivotal role in development and progression of atherosclerotic plaques by virtue of their remarkable plasticity and phenotypic modulation (17-22). VSMCs are recognised as one of the suitable cellular models to detect gene expression signatures underlying molecular processes in atherosclerosis, as well as for functional validation of genes identified/refined through GWAS $(23,24)$.

With the aim to unravel new potential genetic signatures of the atherosclerotic phenotype, we used an integrated approach, joining information from i) gene polymorphisms analysis in a casecontrol study of subjects with or without angiographically demonstrated CAD, ii) microarray-based gene expression analysis on human cultured VSMCs and on carotid artery specimens, and iii) immunohistochemical analysis in carotid artery specimens.

\section{Methods}

A schematic representation of the study design is summarised in Suppl. Figure 1 (available online at www.thrombosis-online.com).

\section{Study population}

This study was performed within the VHS framework, a regional survey designed for identification of new risk factors for CAD in subjects with objective angiographic documentation of their coronary vessels. Details about enrolment criteria have been previously described $(25,26)$. Subjects with proven CAD had at least one of the main epicardial coronary arteries affected (left anterior descending, circumflex, or right) with ( 1 significant stenosis ((50\%). $\mathrm{CAD}$ patients were classified into $\mathrm{MI}$ and non-MI groups on the basis of a thorough review of medical records including history, electrocardiogram, enzyme changes, and/or the typical sequelae of MI on ventricular angiography. CAD-free subjects had completely normal coronary arteries, being submitted to coronary angiography for reasons other than CAD, mainly valvular heart disease. These controls were also required to have neither history nor clinical or instrumental evidence of atherosclerosis in vascular districts beyond the coronary bed. All participants came from the same geographical area (North-East Italy). At the time of blood sampling, a complete clinical history was collected, including the assessment of cardiovascular risk factors such as obesity, smoking, hypertension and diabetes. As regards biochemical analysis, samples of venous blood were withdrawn from each subject, after an overnight fast. A detailed description of laboratory methods is provided in the Suppl. Methods (available online at www.thrombosis-online.com).

The first study population was represented by 510 patients with a history of MI before the age of 65 years and $388 \mathrm{CAD}$-free subjects who were included in the MIGen Consortium as replication population of a GWAS (27). The second study population analysis, addressing specifically the atherosclerotic (more than thrombotic) phenotype, included $442 \mathrm{CAD}$ patients without MI history and 393 CAD-free subjects.

Within the second study population in a subgroup of non-diabetic subjects $(\mathrm{n}=395)$ with available data for fasting insulin concentration, the HOMA (homeostasis model assessment) score was calculated as surrogate of insulin resistance, using the following formula: [fasting glucose $(\mathrm{mmol} / \mathrm{l})^{\star}$ fasting insulin $\left.(\mathrm{mIU} / \mathrm{l}) / 22.5\right]$ (28).

The study was approved by the Ethic Committee of Azienda Ospedaliera Universitaria Integrata, Verona, Italy. Written informed consent was obtained from all the participants after a full explanation of the study.

\begin{tabular}{|c|c|c|c|}
\hline Characteristics & $\begin{array}{l}\text { CAD-free } \\
(n=393)\end{array}$ & $\begin{array}{l}\text { CAD } \\
(n=442)\end{array}$ & $\mathbf{P}$ \\
\hline Age, years & $58.9 \pm 12.1$ & $63.1 \pm 9.1$ & $<0.001$ * \\
\hline Male sex, \% & 62.8 & 72.9 & $0.002 \dagger$ \\
\hline $\mathrm{BMI}, \mathrm{kg} / \mathrm{m}^{2}$ & $25.4 \pm 3.5$ & $26.8 \pm 3.4$ & $<0.001$ * \\
\hline Hypertension, \% & 41.2 & 72.6 & $<0.001 \dagger$ \\
\hline Smoking, \% & 41.9 & 61.3 & $<0.001 \dagger$ \\
\hline Diabetes, $\%$ & 7.1 & 20.4 & $<0.001+$ \\
\hline Glucose, mmol/l & $5.47(5.37-5.58)$ & $5.70(5.58-5.81)$ & $0.007^{*}$ \\
\hline Creatinine, mmol/l & $89.1(86.5-90.9)$ & $90.0(88.2-91.8)$ & $0.587^{*}$ \\
\hline Lipid-lowering therapies, \% & 5.9 & 31.6 & $<0.001 \dagger$ \\
\hline Total cholesterol, mmol/l & $5.44 \pm 1.09$ & $5.45 \pm 1.15$ & 0.904 * \\
\hline LDL-cholesterol, mmol// & $3.50 \pm 0.93$ & $3.62 \pm 0.98$ & 0.098 * \\
\hline HDL-cholesterol, mmol/l & $1.43 \pm 0.42$ & $1.22 \pm 0.32$ & $<0.001$ * \\
\hline Triglycerides, mmol/l & $1.35(1.28-1.40)$ & $1.68(1.62-1.77)$ & $<0.001$ * \\
\hline high sensitivity- C-reactive protein, mg/l & $1.97(1.72-2.27)$ & $3.22(2.83-3.67)$ & $<0.001$ * \\
\hline
\end{tabular}

Table 1: General characteristics of the study populations, with or without coronary artery disease (CAD). 


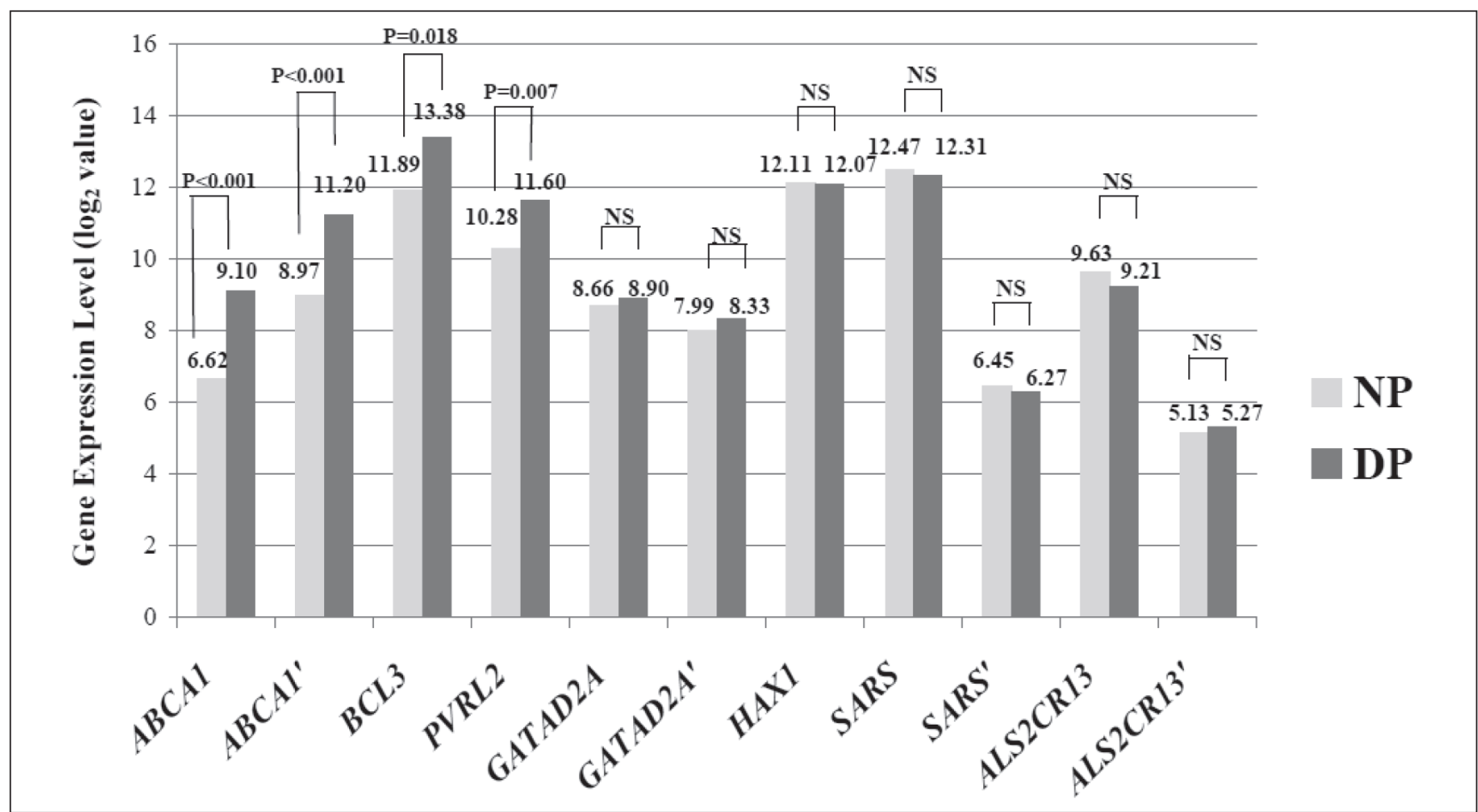

Figure 1: Tissue mRNA expression levels by microarray profiling of atherosclerotic diseased (DP) and non-atherosclerotic (NP) portions of carotid artery. The expression levels of the seven candidate genes, stemming from the preliminary screen in VSMC populations, are reported as log ${ }_{2}$ value. Data obtained with two probes, present in the Agilent microarray, are reported for ABCA1, SARS, GATAD2A and ALS2CR13. NP: grossly non-atherosclerotic carotid portion; DP: atherosclerotic diseased portion; NS: not significant.

\section{SNPs and genotyping}

Genomic DNA was prepared from whole blood samples by phenol-chloroform extraction. The 91 SNPs investigated as replication stage of MIGen study, including intergenic rs10402271 polymorphism (BCL3/PVRL2 locus), were genotyped using the iPLEX MassARRAY platform (Mass Array, Sequenom). PCR primers were designed by Sequenom Mass-Array-Assay-Design program, as previously described (27). The BCL3 polymorphisms, rs2965169 (5' UTR, NM_005178.4: c.-892T>G ) and rs8100239 (intron 1, NM_005178.4: c.256+801T>A) and the PVRL2 polymorphisms, rs3810143 (5' UTR, NM_001042724.1: c.-381T>C or NM_002856.2: c.-381T $>C$ ) and rs1871047 (intron 1, NM_001042724.1: c.88+1876A $>G \quad$ or $\quad$ NM_002856.2: c. $88+1876 \mathrm{~A}>\mathrm{G})$ were genotyped by allele-specific real-time PCR (TaqMan SNP Genotyping Assays, Applied Biosystems, Foster City, CA, USA).

\section{Collection of carotid specimens and histological- immunohistochemical analysis}

Carotid artery specimens were obtained from 58 patients who underwent carotid endarterectomy (CEA) at the Unit of Vascular and Endovascular Surgery of S. Anna University-Hospital (Ferrara, Italy) for extracranial high-grade (>70\%) internal carotid artery stenosis (29) by the same surgeon (FM). Restenotic lesions were excluded. The study was approved by the Ethic Committee of the University-Hospital and written informed consent was obtained from all patients.

Arteriotomy was performed on the common carotid artery and extended to the internal carotid artery (Suppl. Figure 2, available online at www.thrombosis-online.com). The CEA specimen was removed as a single piece and included the atheromatous area and two small adjacent areas, proximal and cranial, respectively, without clear evidence of atherosclerotic lesions. CEA specimen was cut transversally at the bifurcation and the portion towards the aortic arch was used. This segment, consisting of the common carotid artery, was further cut into: a proximal small portion without evidence of atherosclerotic lesions (grossly non-atherosclerotic portion, NP), and the distal one (cephalad), characterised by diffuse atherosclerosis (diseased portion, DP).

Although the NP showed some histological alterations, like a thin thickened intima, this portion was the available and suitable endogenous control.

NP and DP portions were processed for i) histology-immunohistochemistry analysis, ii) total RNA extraction, and iii) VSMC cultures. Portions for total RNA isolation were immediately placed into RNAlater (Ambion Inc., Austin, TX, USA) and then stored at $-80^{\circ} \mathrm{C}$.

Sections of formaldehyde-fixed paraffin-embedded NP and DP were stained with Masson's trichrome for histological analysis (Suppl. Figure 2, available online at www.thrombosis-online.com). 
Immunostaining on paraffin sections was performed as previously described (30) by using the following primary mouse monoclonal antibodies: IgG2a for (-smooth muscle actin ((-SMA, clone 1A4) (31), IgG1 for CD68 (clone KP1; Dako, Glostrup, Denmark) and IgG2a for BCL3 (clone1E8, Abcam, Cambridge, UK). Before using the first antibody, immunoreactivity was intensified by pressure cooker (3 minutes [min]) for BCL3 or microwave treatment (750 $\mathrm{W}, 5 \mathrm{~min}$ ) for $\mathrm{\alpha}$-SMA and CD68.

\section{VSMC culture}

Primary VSMC cultures were obtained from NP and DP carotid specimens collected at surgery, as previously reported (28). After luminal gentle scraping to remove endothelial tissue, the NP (intimal thickening and media) and the DP (plaque with underlying media) were cut into $3 \times 3-\mathrm{mm}$ pieces and plated on separate dishes with the abluminal side in contact with the culture dish. Tissue pieces were cultured in RPMI 1640 medium supplemented with 10\% fetal bovine serum (Invitrogen, Carlsbad, CA, USA), 100 $\mathrm{U} / \mathrm{ml}$ penicillin, $100 \mu \mathrm{g} / \mathrm{ml}$ streptomycin and $2 \mathrm{mM}$ L-glutamine, at $37^{\circ} \mathrm{C}$ with $5 \% \mathrm{CO}_{2}$. Explanted tissues were removed 7-10 days after the first VSMCs appeared. VSMC populations from both NP

Table 2: Distribution of $B C L 3$ and PVRL2 genotypes in the study populations, with or without coronary artery disease (CAD).

\begin{tabular}{|c|c|c|c|}
\hline Genotypes & $\begin{array}{l}\text { CAD-free } \\
(n=393)\end{array}$ & $\begin{array}{l}\text { CAD } \\
(n=442)\end{array}$ & $\mathbf{P}$ \\
\hline \multicolumn{4}{|c|}{ rs2965169 (BCL3) } \\
\hline $\mathrm{TT}$ & 29.3 & 21.8 & \\
\hline TG & 49.4 & 53.1 & 0.020 * \\
\hline GG & 21.4 & 25.2 & \\
\hline \multicolumn{4}{|c|}{ rs8100239 (BCL3) } \\
\hline $\mathrm{TT}$ & 33.1 & 27.1 & \\
\hline $\mathrm{TA}$ & 45.8 & 50.5 & 0.146 * \\
\hline AA & 21.1 & 22.4 & \\
\hline \multicolumn{4}{|c|}{ rs10402271 (intergenic BCL3-PVRL2) } \\
\hline $\mathrm{TT}$ & 50.1 & 46.7 & \\
\hline TG & 41.3 & 41.1 & 0.168 * \\
\hline GG & 8.6 & 12.2 & \\
\hline \multicolumn{4}{|c|}{ rs3810143 (PVRL2) } \\
\hline $\mathrm{TT}$ & 32.5 & 31.7 & \\
\hline $\mathrm{TC}$ & 46.1 & 49.4 & 0.753 * \\
\hline $\mathrm{CC}$ & 21.4 & 18.9 & \\
\hline \multicolumn{4}{|c|}{ rs1871047 (PVRL2) } \\
\hline AA & 36.6 & 33.9 & \\
\hline AG & 45.3 & 46.2 & 0.372 * \\
\hline GG & 18.1 & 19.9 & \\
\hline${ }^{*}$ by $\mathrm{Chi}^{2}$ test $\mathrm{f}$ & ear trend. & & \\
\hline
\end{tabular}

and DP were maintained in identical baseline culture conditions and studied at the third passage. SMC lineage was confirmed by immunofluorescence staining using a mouse IgG2a recognizing (-smooth muscle actin ((-SMA, clone 1A4) (31) and rabbit polyclonal IgGs recognizing both SMMHC types 1 and 2 (BT-562, Biomedical Technologies Inc, Stoughton, MA, USA).

\section{RNA expression study}

Total RNA was isolated either from confluent cultured VSMCs or homogenised NP and DP portions with TRIzol Reagent (Invitrogen) according to the manufacturer's instructions and treated with RNase-free DNase (New England Biolabs, Ipswich, MA, USA). RNA quality was assessed on an Agilent 2100 Bioanalyzer (Agilent Technologies, Palo Alto, CA, USA) and low-quality RNA (RNA integrity number below 7) was excluded from further analyses.

\section{Microarray profiling of cultured VSMCs}

Labelled cRNA was synthesised from $500 \mathrm{ng}$ of total RNA isolated from cultured VSMCs using the Low RNA Input Linear Amplification Kit (Agilent Technologies) in the presence of cyanine 3-CTP (Perkin-Elmer Life Sciences, Boston, MA, USA). Global gene expression was detected using Agilent Whole Human Genome microarray (Cat.No. G4112F, Agilent Technologies), which represents 41,000 unique human transcripts. RNA labelling and hybridisation were performed in accordance to manufacturer's indications. Feature Extraction 10.7 software (Agilent Technologies) was used to obtain the microarray raw-data. Microarray results were analysed using GeneSpring GX 12 software (Agilent Technologies). Data transformation was applied to set all negative raw values at 1.0 , followed by a normalisation on 75 th percentile.

\section{Microarray profiling of whole carotid artery specimens}

RNA from six specimens was hybridised on Agilent whole human genome oligo microarray (Cat.No. G4851A, Agilent Technologies) which represents 60,000 unique human transcripts. RNA labelling and hybridisation were performed in accordance to manufacturer's indications. Feature Extraction software v.10.7 (Agilent Technologies) was used to obtain the microarray raw-data. Microarray results were analysed using the GeneSpring GX 12 software (Agilent Technologies). Data transformation was applied to set all negative raw values at 1.0 , followed by a quantile normalisation.

\section{CDNA preparation and real-time quantitative polyme- rase chain reaction ( $q P C R$ )}

cDNA was obtained from $1 \mu \mathrm{g}$ (cultured cells) or $0.5 \mu \mathrm{g}$ (carotid tissues) of total RNA by reverse transcription using SuperScript VILO cDNA Synthesis Kit (Invitrogen) according to the manufacturer's recommendations. Aliquots of diluted first-strand cDNA were amplified on CFX96 Real-Time PCR Detection System (BioRad, Hercules, CA, USA) using SsoFast EvaGreen Supermix (Bio- 
Figure 2: Association between the carriership of BCL3 rs2965169 minor allele $G$ and coronary artery disease (CAD). The strength of the association was estimated by means of ORs with $95 \% \mathrm{Cl}$ at univariate, sex- and age-adjusted, and full adjusted analysis. The homozygotes for the major allele (TT) were considered as reference. * by logistic regression analysis adjusted for sex, age, BMI, smoking, hypertension, diabetes, LDL- and HDL-cholesterol, triglycerides, creatinine, and hs-CRP.

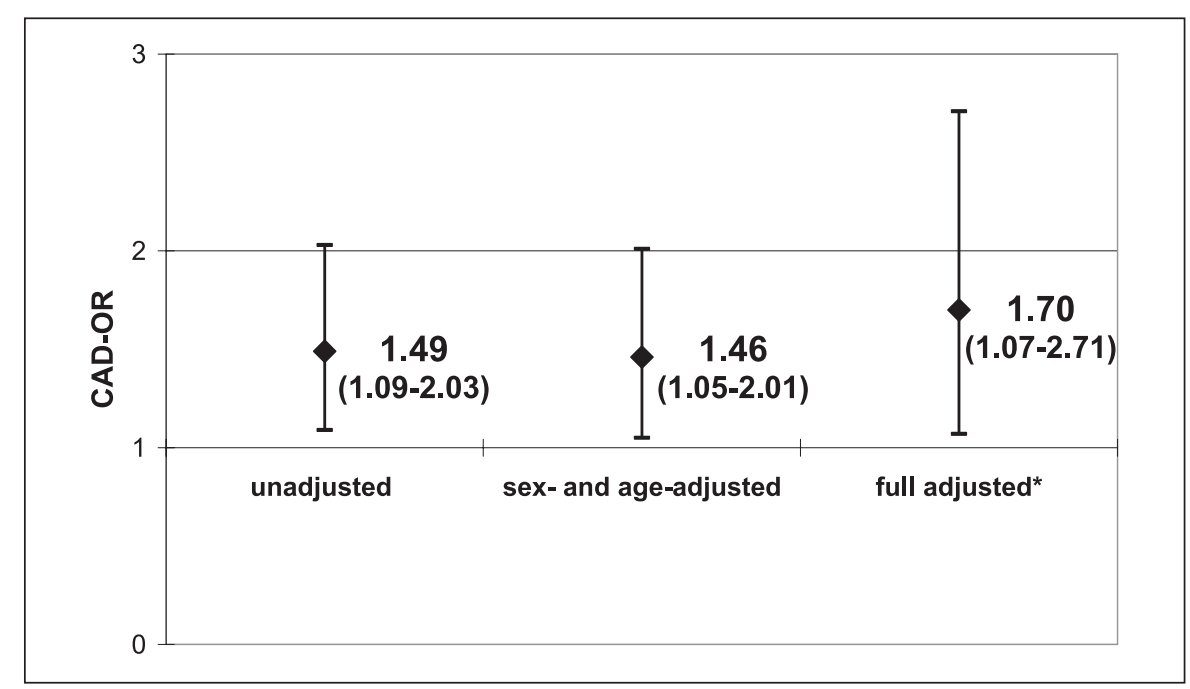

Rad). PCR protocol was: $95^{\circ} \mathrm{C}$ for 30 seconds (sec), then 40 cycles of 5 secat $95^{\circ} \mathrm{C}$ and $10 \mathrm{sec}$ at $60^{\circ} \mathrm{C}$.

Forward and reverse primers are reported in the Suppl. Table 1 (available online at www.thrombosis-online.com). Each reaction was performed in triplicate. The relative levels of mRNAs were calculated by the comparative CT method by using $18 \mathrm{~S}$ rRNA as endogenous control in cell populations or ACTB in carotid specimens. Values were expressed as mean fold change \pm standard error of the mean (SEM).

\section{Statistical analysis}

Differences in mRNA expression levels between VSMC populations by microarray-based transcriptome analysis were evaluated by using the $\log$-transformed $\left(\log _{2}\right)$ ratio values (DP/NP) and assuming the $\log$ ratio equal to 0 when there is no difference of expression (32). A p-value $<0.05$ was considered significant without correction for multiple testing. Relationships between the foldchange $\left(\log _{2} \mathrm{DP} / \mathrm{NP}\right.$ ratio) and the p-value (negative $\left.\log _{10}\right)$ were reported in a volcano plot.

Genes differentially expressed between three atherosclerotic and three non-atherosclerotic carotid specimens in microarray experiment were identified using GeneSpring GX software. A filter on fold change (1.5 was applied to the list of probes expressed in at least one sample. Then, a moderated t-test was used to identify the significantly modulated genes with $\mathrm{p}<0.05$ and false discovery rate $(\mathrm{FDR})=10 \%$. Gene expression levels between VSMC populations or carotid portions in q-PCR analysis were compared by means of paired or unpaired t-test.

As regards population data, distributions of continuous variables in groups were expressed as means \pm standard deviation (SD). Statistical analysis on skewed variables, like glucose, creatinine, triglyceride, and high-sensitivity C-reactive protein (hsCRP), was computed on the corresponding log-transformed values. Thus, results are reported as geometric mean with $95 \%$ confidence interval (95\% CI). Quantitative data were assessed using the Student's t-test or analysis of variance (ANOVA), with polynomial contrast for linear trend when indicated. Qualitative data were analysed with either $\mathrm{Chi}^{2}$-test or $\mathrm{Chi}^{2}$ for linear trend analysis when indicated. Within each group examined, the frequencies of the genotypes associated with each of the polymorphisms were compared by the $\mathrm{Chi}^{2}$-test with the values predicted on the basis of the Hardy-Weinberg equilibrium.

In the first case-control population the strength of association with CAD/MI was assessed by means of a sex- and age-adjusted model. In the second case-control population the strength of association with $\mathrm{CAD}$ was estimated calculating the odds ratios (ORs) with $95 \%$ CIs by multiple logistic regression after sex- and age-adjustment and then after adjustment for all the traditional cardiovascular risk factors (i.e. sex, age, body mass index [BMI], smoking, hypertension, diabetes, LDL- and HDL-cholesterol, triglycerides, creatinine, and hs-CRP). A value of $\mathrm{p}<0.05$ was considered statistically significant. Statistical power was estimated by Altman nomogram (33). Calculations were performed with IBM SPSS 20.0 statistical package (IBM Inc., Armonk, NY, USA).

Haplotype frequencies were estimated using the $\mathrm{R}$ software with haplo.stats package (R Foundation for Statistical Computing, Vienna, Austria; http: //www.R-project.org). The associations between haplotypes and laboratory and clinical outcomes were examined using a generalised linear model regression of a trait on haplotype effects, allowing for ambiguous haplotypes (haplo.glm function) (34). Statistical significance of associations was ascertained by randomly permuting the disease status in 1,000 replicates by Monte Carlo method.

\section{Results}

\section{SNPs selection}

Starting from a previous analysis of 91 SNPs in 898 subjects of the VHS population (388 CAD-free and $510 \mathrm{CAD}$ with MI) within stages of replication of GWAS - MIGen - for cardiovascular risk (27), we selected 15 SNPs showing nominally an association with MI by means of a sex- and age-adjusted model with $P$ values arbit- 
rarily set at $<0.10$ (Suppl. Table 2, available online at www.thrombo sis-online.com).

As more than half $(8 / 15)$ of the selected SNPs localise within intergenic regions, the genomic regions tagged by the 15 SNPs were examined in the NCBI database for the presence of validated or putative coding gene sequences within a region of $400 \mathrm{~kb}$ centred on each SNP. This search identified 77 genes, listed in Suppl. Table 3 (available online at www.thrombosis-online.com).

\section{Transcriptome analysis of cultured VSMCs and of carotid specimens}

As a preliminary screen to identify, among the 77 genes, candidates for further evaluation, we performed a microarray-based transcriptome analysis of VSMC populations, cultured from NP: DP couples collected from three patients. The Agilent whole human genome microarray (44k) included probes for 71 of the 77 neighbouring genes. The analysis revealed significant differences $(\mathrm{P}<0.05)$ in the expression levels for seven genes $(A B C A 1$, ALS2CR13, PVRL2, BCL3, HAX1, SARS, GATAD2A, Suppl.Table 4 and Suppl. Figure 3, both available online at www.thrombosis-on line.com). Validation was conducted by qPCR analyses in independent cell populations (see Suppl. Results, available online at www.thrombosis-online.com).

To explore also in the vessel wall the differences in the expression levels detected in cultured VSMCs, we performed a second microarray experiment of whole atherosclerotic and nonatherosclerotic specimens, collected from four additional patients. The comparison (fold change $(1.5, \mathrm{p}<0.05$, FDR $10 \%)$ of DP and NP tissues showed that three (ABCA1, BCL3 and PVRL2) out the seven genes, identified in the first transcriptome screen, were significantly up regulated in DP ( $>$ Figure 1$)$. This analysis revealed the most significant differences for the ATP-binding membrane cassette transporter A1 (ABCA1), whose role in lipid metabolism and in atherosclerosis pathways is well recognised.

We addressed then our attention to the BCL 3 and PVRL2 genes, contiguously located on the chromosome region (19q13) marked by the intergenic rs10402271. With the aim to validate findings from microarray profiling of specimens, qPCR analyses were performed on carotid tissue samples from independent patients. BCL3 mRNA levels were higher in DP than in NP specimens by comparison of i) DP: NP couples (five patients), with a mean fold change difference of $10.07 \pm 2.87(\mathrm{p}=0.034)$ as well as ii) noncoupled DP and NP (14 patients), with a mean fold change difference of $6.81 \pm 3.16(p=0.013)$. PVRL2 expression levels were significantly higher in DP by comparison of couples (mean fold change $2.60 \pm 0.46, \mathrm{p}=0.026$ ) and, as a trend, in non- coupled DP and NP (mean fold change $2.52 \pm 0.80 ; \mathrm{p}=0.060$ ).

\section{$B C L 3$ SNPs genotyping for association with CAD}

As the originally investigated rs10402271 maps in the intergenic $19 \mathrm{q} 13$ region between $B C L 3$ and $P V R L 2$, intragenic SNPs, two for $B C L 3$ (rs2965169 and rs8100239, previously associated to oral clefts) (35), and two for PVRL2 (rs3810143 and rs1871047), were selected for the second genetic association analysis. It is worthy to note that the low MAF value $(<0.02$, by NCBI) of cDNA SNPs, both in BCL3 and PVRL2, makes them not eligible for this study.

We compared CAD-free controls $(n=393)$ with a second population of CAD patients without previous MI $(n=442)$. This cohort enabled us to preferentially investigate the atherosclerotic phenotype, and to avoid the potential biases due to selection/survival related to MI history.

The clinical and laboratory characteristics of the two groups are summarised in $>$ Table 1 , while the data about genotype prevalence of the five SNPs are reported in Table 2. Genotypes had a distribution consistent with Hardy-Weinberg equilibrium in both cases and controls and showed a low or moderate degree of linkage disequilibrium (Suppl. Figure 4, available online at www.thrombo sis-online.com), which were in accordance with Hap Map data.

While the PVRL2 rs3810143 and rs1871047 and the intergenic rs10402271 did not show a significant association with CAD, the $B C L 3$ rs2965169 genotype distribution differed significantly between cases and controls ( $>$ Table 2). The carriership of the minor
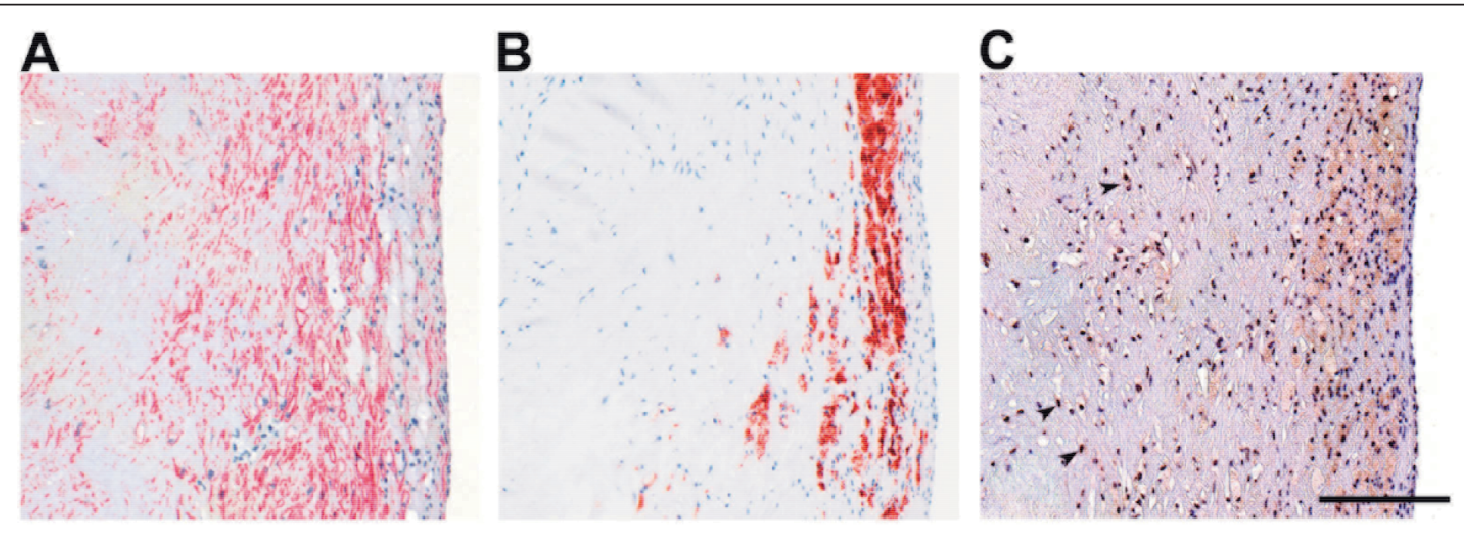

Figure 3: BCL3 protein expression in atherosclerotic (DP) carotid specimens. Representative immunohistochemical staining of the DP plaque for a-SMA (A), CD68 (B) and BCL3 (C). BCL3 is expressed by plaque foam cells and rare VSMCs (arrows). The lumen is located on the right side of the pictures. Bar $=200 \mu \mathrm{m}$. 
Table 3: Distribution of $B C L 3$ haplotypes in the whole study population and in subjects with or without coronary artery disease (CAD).

\begin{tabular}{l|l|l|l|l|l}
\hline & BCL3 polymorphisms & \multicolumn{3}{l}{ Haplotype frequency (\%) } \\
\cline { 2 - 7 } & rs2965169 & rs8100239 & $\begin{array}{l}\text { Whole study } \\
\text { population } \\
(\mathbf{n = 8 3 5 )}\end{array}$ & $\begin{array}{l}\text { CAD-free } \\
\text { subjects } \\
(\mathbf{n = 3 9 3 )}\end{array}$ & $\begin{array}{l}\text { CAD } \\
\text { patients } \\
(\mathbf{n}=442)\end{array}$ \\
\hline Haplotype 1 & T & T & 46.9 & 49.3 & 44.9 \\
\hline Haplotype 2 * & G & A & 41.9 & 39.2 & 44.3 \\
\hline Haplotype 3 & G & T & 7.1 & 6.7 & 7.4 \\
\hline Haplotype 4 & T & A & 4.1 & 4.8 & 3.4 \\
\hline * subjects with G-A haplotype carried a greater risk of CAD than subjects with T-T haplotype (OR 1.25 \\
with 95\%Cl 1.02-1.53, p=0.035; p=0.026 in a sex- and age-adjusted model after 1,000 permutations \\
by the Monte Carlo method).
\end{tabular}

allele $\mathrm{G}$ was more frequent in CAD patients ( $\$$ Table 2) and remained independently associated with CAD in different adjusted logistic regression models ( $\$$ Figure 2).

The carriership of the BCL3 rs8100239 minor allele A appeared to be more represented in CAD patients, but did not reach a statistically significant difference $(72.9 \%$ vs $66.9 \%, \mathrm{p}=0.062)$. Further analyses showed association between rs8100239 and some intermediate metabolic phenotypes, with the A allele linked with insulin resistance-related conditions (see Suppl. Results and Suppl. Tables 5A and 6, all available online at www.thrombosis-online. com). On the other hand, associations of BCL3 rs2965169 (Suppl. Table 5B, available online at www.thrombosis-online.com), PVRL2 rs3810143 and rs1871047, and intergenic rs10402271 genotypes (data not shown) with lipid profile and metabolic phenotypes were not detected.The haplotype analysis based on BCL3 rs2965169 and rs8100239 showed that the G-A haplotype, containing the minor alleles, was more represented in patients with CAD than in CADfree ( Table 3, OR 1.25 with 95\%CI 1.02-1.53 as compared with the most frequent $\mathrm{T}-\mathrm{T}$ haplotype; $\mathrm{p}=0.035$ ).

\section{Expression of the BCL3 protein in carotid specimens}

The expression of BCL3 protein was investigated in five NP and 10 DP specimens by immunohistochemical analysis. A positive staining for BCL3 ( Figure 3) was found in $20-40 \%$ of plaque foam cells and in rare intimal and medial VSMCs in nine of $10 \mathrm{DP}$ samples. We did not detect any BCL3-expressing cells in the intima-media of all the NP samples (data not shown).

\section{Discussion}

GWAS are powerful tools for unveiling the genetic components of $\mathrm{CAD}$ and MI, but their rigorous approach may hide some potential limitations which should be taken into account. The extensive multiple testing correction in GWAS, necessary to exclude falsepositive results, may simultaneously discard genetic loci which could contribute to pathophysiology (12-14). Moreover, the considerable overlap among clinical phenotypes of CVD, leads several GWAS to cumulate indifferently CAD and MI in their analysis (3).
Recent studies have consistently demonstrated that some SNPs are associated more with CAD than with a specific predisposition to MI (36). Combined approaches exploiting complementary methodologies may help to overcome these limitations and lead to significant advances in understanding the mechanisms of multifactorial diseases, like CVD (37-39).

Our experimental design integrates analyses at DNA, RNA and protein levels.The GWAS-related tag SNPs and two transcriptome analyses, in both cultured cells and tissue, enabled us to propose three candidate genes, BCL3, PVRL2 and ABCA1. The last one has been extensively investigated in relation to the pathogenetic mechanisms of atherosclerotic vascular diseases (40).The recognised role of $A B C A 1$ in CVD, strengthened by the observation that $A B C A 1$ mutations cause Tangier disease and premature atherosclerosis (41), supports our experimental approach aimed at detecting genetic components of atherosclerotic vascular diseases.

The two other candidate genes (BCL3 and PVRL2) were contiguously located on chromosome 19 , in a region previously associated to plasma lipid traits (42), thus increasing the interest for this genomic region. This prompted us to further investigate specific SNPs for BCL3 and the adjacent PVRL2, in a second casecontrol analysis considering CAD patients without MI history. This step produced coherent results: the carriership of BCL3 rs $2965169 \mathrm{G}$ allele was associated to an increased risk of CAD independently from traditional atherosclerosis risk factors and the $B C L 3$ rs8100239 A allele, in moderate linkage, tended also to be more represented among $\mathrm{CAD}$ patients. Consistently the rs2965169 G - rs8100239 A haplotype was more frequent in CAD patients than in CAD-free subjects.

Although until now classical GWAS approaches have failed to identify $B C L 3$ as risk gene for CAD (1), a recent study combining GWAS results with expression quantitative trait loci (eQTLs) analysis identified $B C L 3$ as a potential new risk gene for Crohn's disease (43), a chronic inflammatory disease associated with an increased atherosclerotic risk unexplained by traditional cardiovascular risk factors (44). With regard to the results at RNA level, our findings are also consistent with data from a transcriptome analysis by microarray on human carotid plaques (GEO database, GSE 43292), which shows higher BCL3 mRNA levels in the majority of 
atheroma plaques as compared to the macroscopically intact tissues.

Evidence from our study for BCL3 protein expression in atherosclerotic vascular vessel wall, not reported so far, further supports the hypothesis of a role of BCL3 in CVD and fit with RNA data in carotid specimens. We found BCL3 positivity only in atherosclerotic portions and in cells, like foam cells and VSMCs, which are well known to play crucial role in atherogenesis. Actually, we observed very few positive VSMCs, but this result is not surprising taking into account the lack of BCL3 positivity in most of tissues as reported in the Human Protein Atlas database. On the other hand, the very low BCL3 protein expression levels in diseased tissue might not favour to disentangle the interpretation of BCL3 role in atherosclerosis.

BCL3 is a transcriptional coregulator and member of the inhibitor of nuclear factor- $\mathrm{KB}$ (IкB) family, which takes part to both positive and negative modulation of genes belonging to several pathways, including those of cell death/proliferation, inflammatory and stress responses $(45,46)$. Biological information from Gene Ontology database locates BCL3 in multiple processes which are known to be relevant in atherosclerosis (Suppl. Figure 5, available online at www.thrombosis-online.com).

BCL3 was also proposed as a key interface for regulatory crosstalk between inflammation and cellular energy metabolism (47). According with this hypothesis, in our study the BCL3 rs8100239 A allele correlated with traits usually characterizing the so-called metabolic syndrome, a still debated cluster of risk factors including altered energy metabolism and whose core is thought to be insulin resistance $(48,49)$. The lack of waist circumference data in our population does not allow to define the conventionally stated diagnosis of metabolic syndrome (50). Nonetheless, we observed significant associations with both unfavorable lipid profile and overweight. Although assessed only in a relatively small group of subjects, the A allele was associated also with both hyperinsulinaemia and higher HOMA score, which is considered a surrogate measure of insulin resistance.

\section{What is known about this topic?}

- The stringent level of statistical significance required in genomewide association studies may lead to discard genetic variants that could contribute to disease risk.

- Integrated genomic and transcriptomic investigations might be a tool to unravel new genetic signatures for cardiovascular disease.

- The expression of the proto-oncogene BCL3 in human atherosclerotic lesions has not previously been reported.

\section{What does this paper add?}

- BCL3 mRNA and protein were differently expressed in atherosclerotic versus non-atherosclerotic portions of carotid artery.

- $B C L 3$ genotypes were associated with both coronary artery disease and metabolic derangements.

- The proto-oncogene BCL3 may play a role in atherosclerosis.
Our study has some limitations that warrant discussion. The allele specific contribution to BCL3 mRNA expression level, which could provide insight for a functional effect and for the associated risk, is difficult to determine because of the SNPs localisation (5' UTR and intron 1) and the virtual absence of in linkage coding SNPs. On the other hand, at inspection (UCSC Genome Browser, hg19, ENCODE data) for functional elements by H3K27 histone acetylation mark and by chromatin state segmentation analysis, both BCL3 rs2965169 and rs8100239 polymorphisms have been shown to lie within potentially active regulatory elements in endothelial-, B-lymphocyte- and fibroblast cell lines, which are of interest for atherosclerosis pathways.

The transcriptional analysis was obtained in VSMC populations or in whole specimens from a small number of patients. This limitation has been tackled by i) the study of couples of specimens from the same donor, which would reduce variability in the gene expression profile; ii) the combination of subsequent and independent transcriptome analyses, expected to increase robustness of the approach and to reveal hits of "in vivo" relevance; and iii) the validation of transcriptome changes by qPCR. We also disclose that the complex transcriptional and post-transcriptional regulation of $B C L 3$ (45) could be perturbed by in vitro culture state and thus differ from the in vivo condition.

Regarding the statistical analysis, the lack of adjustment for multiple comparisons is a further limitation of our work. We attempted to overcome this drawback not only by requiring casecontrol analyses in two CAD populations (with or without MI), but also by showing additional support for the association between BCL3 and atherosclerosis at mRNA (in cultured VSMC populations and in carotid tissues) and protein levels.

Other significant caveats of this study are related to the population analysis, including the retrospective case-control design, the relatively low number of subjects, and the lack of some clinical data. On the other hand, a remarkable strength of this study is represented by the angiography-evaluation of the coronary artery bed, which allows a clear-cut definition of the clinical phenotype and avoids the possibility to include in the control group subjects with subclinical, but significant CAD. Finally, considering the different prevalence of the carriership of rs $2965169 \mathrm{G}$ allele among subjects with or without $\mathrm{CAD}$ in the second case-control analysis, the statistical power of our study was about $80 \%$ by Altman nomogram with a significance level of 0.05 (33).

In summary, our integrated approach suggests for the first time the involvement of BCL3 in CVD, which could be partly mediated through the influence on metabolic phenotypes. Our results should be considered as hypothesis-generating and further epidemiological studies on larger samples, as well as basic investigations about BCL3 expression and function, are needed to confirm the role of BCL3 in the pathways of atherosclerosis.

\section{Acknowledgements}

We are very grateful to Prof. Sekar Kathiresan (Cardiovascular Research Center and Cardiology Division and Center for Human Genetic Research, Massachusetts General Hospital, Boston, Program in Medical and Population Genetics, Broad Institute of MIT 
and Harvard, Cambridge, Massachusetts 02142, USA) for the ongoing cooperation and for providing the data about SNPs of MIGen Consortium. We wish to thank Mrs. Maria Zoppi for her invaluable secretary help, and Dr. Patrizia Guarini, Diego Minguzzi and Patrizia Pattini for their excellent technical help.

\section{Conflicts of interest}

None declared.

\section{References}

1. Kathiresan S, Srivastava D. Genetics of human cardiovascular disease. Cell 2012; 148: 1242-1257.

2. Roger VL, Go AS, Lloyd-Jones DM, et al. American Heart Association Statistics Committee and Stroke Statistics Subcommittee. Executive summary: heart disease and stroke statistics-2012 update: a report from the American Heart Association. Circulation 2012; 125: 188-197.

3. Ding K, Kullo IJ. Genome-wide association studies for atherosclerotic vascular disease and its risk factors. Circ Cardiovasc Genet 2009; 2: 63-72.

4. O'Donnell CJ, Nabel EG. Genomics of cardiovascular disease. N Engl J Med 2011; 365: 2098-2109.

5. Bijnens APJJ, Lutgens E, Ayoubi T, et al. Genome-wide expression studies of atherosclerosis-Critical issue in methodology, analysis, interpretation of transcriptomics data. Arterioscler Thromb Vasc Biol 2006; 26: 1226-1235.

6. Miller DT, Ridker PM, Libby P, et al. Atherosclerosis: the path from genomics to therapeutics. J Am Coll Cardiol 2007; 49: 1589-1599.

7. Cappola TP, Margulies KB. Functional genomics applied to cardiovascular medicine. Circulation 2011; 124: 87-94.

8. Park SW, Moon YA, Horton JD. Post-transcriptional regulation of low density lipoprotein receptor protein by proprotein convertase subtilisin/kexin type 9a in mouse liver. J Biol Chem 2004; 279: 50630-50638.

9. Lambert G, Charlton F, Rye KA, et al. Molecular basis of PCSK9 function. Atherosclerosis 2009; 203: 1-7.

10. Musunuru K, Strong A, Frank-Kamenetsky M, et al. From noncoding variant to phenotype via SORT1 at the 1p13 cholesterol locus. Nature 2010; 466: 714-719.

11. Yang $X$. Use of functional genomics to identify candidate genes underlying human genetic association studies of vascular diseases. Arterioscler Thromb Vasc Biol 2012; 32: 216-222

12. Marian AJ, Belmont J. Strategic approaches to unravelling genetic causes of cardiovascular diseases. Circ Res 2011; 108: 1252-1269.

13. Stahl EA, Wegmann D, Trynka G, et al. Bayesian inference analyses of the polygenic architecture of rheumatoid arthritis. Nat Genet 2012; 44: 483-489.

14. Park JH, Wacholder S, Gail MH, et al. Estimation of effect size distribution from genome-wide association studies and implications for future discoveries. Nat Genet 2010; 42: 570-575.

15. Ross R. Mechanisms of disease: Atherosclerosis-An inflammatory disease. N Engl J Med 1999; 340: 115-126.

16. Lusis AJ. Atherosclerosis. Nature 2000; 407: 233-241.

17. Campbell JH, Campbell GR. Smooth Muscle Phenotypic Modulation-A Personal Experience. Arterioscler Thromb Vasc Biol 2012; 32: 1784-1789.

18. Gomez D, Owens GK. Smooth muscle cell phenotypic switching in atherosclerosis. Cardiovasc Res 2012; 95: 156-164.

19. Hao H, Gabbiani G, Bochaton-Piallat ML. Arterial smooth muscle cell heterogeneity: implications for atherosclerosis and restenosis development. Arterioscler Thromb Vasc Biol 2003; 23: 1510-1520.

20. Doran AC, Meller N, McNamara CA. Role of smooth muscle cells in the initiation and early progression of atherosclerosis. Arterioscler Thromb Vasc Biol 2008; 28: 812-819.

21. Lacolley P, Regnault V, Nicoletti A, et al. The vascular smooth muscle cell in arterial pathology: a cell that can take on multiple roles. Cardiovasc Res 2012; 95: 194-204.

22. Johnson JL. Emerging regulators of vascular smooth muscle cell function in the development and progression of atherosclerosis. Cardiovasc Res 2014; Epub ahead of print
23. Motterle $\mathrm{A}, \mathrm{Pu} \mathrm{X}$, Wood $\mathrm{H}$, et al. Functional analyses of coronary artery disease associated variation on chromosome 9p21 in vascular smooth muscle cells. Hum Mol Genet 2012; 21: 4021-4029.

24. Perisic L, Hedin E, Razuvaev A, et al. Profiling of atherosclerotic lesions by gene and tissue microarrays reveals PCSK6 as a novel protease in unstable carotid atherosclerosis. Arterioscler Thromb Vasc Biol 2013; 33: 2432-2443.

25. Bozzini C, Girelli D, Bernardi F, et al. Influence of polymorphisms in the factor VII gene promoter on activated factor VII levels and on the risk of myocardial infarction in advanced coronary atherosclerosis. Thromb Haemost 2004; 92: 541-549.

26. Martinelli N, Girelli D, Lunghi B, et al. Polymorphisms at LDLR locus may be associated with coronary artery disease through modulation of coagulation factor VIII activity and independently from lipid profile. Blood 2010; 116: 5688-5697.

27. Kathiresan S, Myocardial Infarction Genetics Consortium. Genome-wide association of early-onset myocardial infarction with single nucleotide polymorphisms and copy number variants. Nat Genet 2009; 41: 334-341.

28. Matthews DR, Hosker JP, Rudenski AS, et al. Homeostasis model assessment: insulin resistance and beta-cell function from fasting plasma glucose and insulin concentrations in man. Diabetologia 1985; 28: 412-419.

29. North American Symptomatic Carotid EndarterectomyTrial Collaborators. Beneficial effect of carotid endarterectomy in symptomatic patients with highgrade carotid stenosis. N Engl J Med 1991; 325: 445-453.

30. Coen M, Marchetti G, Palagi PM, et al. Calmodulin expression distinguishes the smooth muscle cell population of human carotid plaque. Am J Pathol 2013; 183: 996-1009.

31. Skalli O, Ropraz P, Trzeciak A, et al. A monoclonal antibody against alphasmooth muscle actin: a new probe for smooth muscle differentiation. J Cell Biol 1986; 103: 2787-2796.

32. Cui X, Churchill GA. Statistical tests for differential expression in cDNA microarray experiments. Genome Biol 2003; 4: 210-219.

33. Altman DG. Statistics and ethics in medical research. How large a sample? $\mathrm{Br}$ Med J 1980; 281: 1336-1338.

34. Lake SL, Lyon H, Tantisira K, et al. Estimation and tests of haplotype-environment interaction when linkage phase is ambiguous. Hum Hered 2003; 55: 56-65.

35. Park BY, Sull JW, Park JY, et al. Differential parental transmission of markers in BCL3 among Korean cleft case-parent trios. J Prev Med Public Health 2009; 42: $1-4$.

36. Reilly MP, Li M, He J, et al. Myocardial Infarction Genetics Consortium; Wellcome Trust Case Control Consortium. Identification of ADAMTS7 as a novel locus for coronary atherosclerosis and association of $\mathrm{ABO}$ with myocardial infarction in the presence of coronary atherosclerosis: two genome-wide association studies. Lancet 2011; 377: 383-392.

37. Folkersen L, van't Hooft F, Chernogubova E, et al. BiKE and ASAP study groups. Association of genetic risk variants with expression of proximal genes identifies novel susceptibility genes for cardiovascular disease. Circ Cardiovasc Genet 2010; 3: 365-373

38. Ramsey SA, Gold ES, Aderem A. A systems biology approach to understanding atherosclerosis. EMBO Mol Med 2010; 2: 79-89.

39. Maouche S, Schunkert H. Strategies beyond genome-wide association studies for atherosclerosis. Arterioscler Thromb Vasc Biol 2012; 32: 170-181.

40. Oram JF, Vaughan AM. ATP-Binding cassette cholesterol transporters and cardiovascular disease. Circ Res 2006; 99: 1031-1043.

41. Fitzgerald ML, Mujawar Z, Tamehiro N. ABC transporters, atherosclerosis and inflammation. Atherosclerosis 2010; 211: 361-370.

42. Talmud PJ, Drenos F, Shah S, et al. Gene-centric association signals for lipids and apolipoproteins identified via the Human CVD BeadChip. Am J Hum Genet 2009; 85: 628-642.

43. Fransen K, Visschedijk MC, van Sommeren S, et al. Analysis of SNPs with an effect on gene expression identifies UBE2L3 and BCL3 as potential new risk genes for Crohn's disease. Hum Mol Genet 2010; 19: 3482-3488.

44. Miller AM, McInnes IB. Cytokines as therapeutic targets to reduce cardiovascular risk in chronic inflammation. Curr Pharm Des 2011; 17: 1-8.

45. Palmer S, Chen YH. Bcl-3, a multifaced modulator of NF-kB-mediated gene transcription. Immunol Res 2008; 42: 210-218.

46. Hinz M, Arslan SÇ, Scheidereit C. It takes two to tango: IкBs, the multifunctional partners of NF- $\kappa$ B. Immunol Rev 2012; 246: 59-76.

47. Yang J, Williams RS, Kelly DP. BCL3 interacts cooperatively with peroxisome proliferator-activated receptor gamma (PPARgamma) coactivator lalpha to 
coactivate nuclear receptors estrogen-related receptor alpha and PPARalpha. Mol Cell Biol 2009; 29: 4091-4102.

48. Alberti KG, Eckel RH, Grundy SM, et al. Harmonizing the metabolic syndrome: a joint interim statement of the International Diabetes Federation Task Force on Epidemiology and Prevention; National Heart, Lung, and Blood Institute; American Heart Association; World Heart Federation; International Atherosclerosis Society; and International Association for the Study of Obesity. Circulation 2009; 120: 1640-1645.
49. Grundy SM. Metabolic syndrome: a multiplex cardiovascular risk factor. J Clin Endocrinol Metab 2007; 92: 399-404.

50. Executive Summary of The Third Report of The National Cholesterol Education Program (NCEP) Expert Panel on Detection, Evaluation, And Treatment of High Blood Cholesterol In Adults (Adult Treatment Panel III). J Am Med Assoc 2001; 285: 2486-2497. 